\title{
Neural aspects of cohort-size reduction during visual gating
}

Citation for published version (APA):

Bles, M., Alink, A., \& Schmitt, B. M. (2007). Neural aspects of cohort-size reduction during visual gating. Brain Research, 1150, 143-154. https://doi.org/10.1016/j.brainres.2007.02.071

Document status and date:

Published: 01/01/2007

DOI:

10.1016/j.brainres.2007.02.071

Document Version:

Publisher's PDF, also known as Version of record

Document license:

Taverne

Please check the document version of this publication:

- A submitted manuscript is the version of the article upon submission and before peer-review. There can be important differences between the submitted version and the official published version of record.

People interested in the research are advised to contact the author for the final version of the publication, or visit the DOI to the publisher's website.

- The final author version and the galley proof are versions of the publication after peer review.

- The final published version features the final layout of the paper including the volume, issue and page numbers.

Link to publication

\footnotetext{
General rights rights.

- You may freely distribute the URL identifying the publication in the public portal. please follow below link for the End User Agreement:

www.umlib.nl/taverne-license

Take down policy

If you believe that this document breaches copyright please contact us at:

repository@maastrichtuniversity.nl

providing details and we will investigate your claim.
}

Copyright and moral rights for the publications made accessible in the public portal are retained by the authors and/or other copyright owners and it is a condition of accessing publications that users recognise and abide by the legal requirements associated with these

- Users may download and print one copy of any publication from the public portal for the purpose of private study or research.

- You may not further distribute the material or use it for any profit-making activity or commercial gain

If the publication is distributed under the terms of Article $25 \mathrm{fa}$ of the Dutch Copyright Act, indicated by the "Taverne" license above, 


\title{
Research Report
}

\section{Neural aspects of cohort-size reduction during visual gating}

\author{
Mart Bles $^{a, *}$, Arjen Alink ${ }^{a, b}$, Bernadette M. Jansma ${ }^{a}$ \\ ${ }^{a}$ Maastricht University, Faculty of Psychology, department of Cognitive Neuroscience, PO Box 616, 6200 MD Maastricht, The Netherlands \\ ${ }^{\mathrm{b}} \mathrm{Max}$ Planck Institute for Brain Research, Frankfurt, Germany
}

\section{A R T I C L E I N F O}

Article history:

Accepted 27 February 2007

Available online 3 March 2007

Keywords:

Cohort

Coactivation

Inhibition

Lexicon

Gating

ERP

\begin{abstract}
A B S T R A C T
In psycholinguistics, the coactivation of lexical candidates by partial input has received a lot of attention. More recently, psychophysiological studies have shed more light on this issue and identified two possible time windows in which effects of coactivation of lexical candidates can be observed in event-related potentials (ERPs). However, these studies cannot be used to disentangle effects of coactivation from the inhibition of candidates that do not match input. A new visual gating paradigm is presented, in which stimuli were visually presented letter by letter, decreasing the size of the cohort of lexical candidates as more letters are presented. Stimuli were created such that at the letter position of interest, the amount of coactivated candidates was kept constant while manipulating the size of the reduction of the cohort. The resulting ERPs indicate two components (frontal P2, and a left temporal late negativity) that scaled with cohort-size reduction. These results show that a visual gating paradigm can be used to disentangle coactivation of lexical candidates from inhibition of non-matching items, and that these processes are closely related to each other in time.
\end{abstract}

C 2007 Elsevier B.V. All rights reserved.

\section{Introduction}

It is generally assumed that, in language comprehension, partial auditory or visual input activates cohorts of lexical candidates that match this input. With more incoming information, the initial set of candidates is reduced such that only matching items remain in the cohort. The reduction process terminates when one candidate remains, at which point word recognition takes place. Cognitive models of speech and written language comprehension deal with acoustic, graphic, phonetic and phonological encoding, accompanied by semantic and syntactic activation and discourse integration (e.g., Ans et al., 1998; Coltheart et al., 1991; Cutler and Clifton, 1999; Gaskell and Marslen-Wilson, 1997; Goldinger et al., 1992; Johnson and Pugh, 1994; MarslenWilson, 1987; Masson, 1995; McClelland and Elman, 1986;
Norris et al., 1995; Perfetti, 1999). Such models have elaborated ideas about how the activation of the initial set and the selection of the winner actually take place. These ideas differ between models, and there is still ongoing discussion about the details of word recognition. A consistent idea across the literature is that partial linguistic input can coactivate a set of several lexical items (hereafter called a 'cohort'). Coactivation of lexical items has received a lot of attention in psycholinguistics and more recently in the psychophysiological literature. To our knowledge, less work has been reported on the reduction of the cohort of activated items and the nature of the neural selection of the target word from this cohort. The goal of this paper is to focus on processes related to neural aspects of "cohort-size reduction" during word recognition.

Evidence for the coactivation of lexical candidates can be found in a variety of studies with different psycholinguistic

\footnotetext{
* Corresponding author. Fax: +31 433884125.

E-mail address: M.Bles@psychology.unimaas.nl (M. Bles).
} 
paradigms. In cross-modal semantic priming studies, gated fragments of words such as captain are followed by a visually presented target word for lexical decision. The target word can be semantically related either to the spoken word itself (e.g., ship, semantically related to captain) or to a cohort competitor sharing the same initial sounds (e.g., guard, semantically related to captive). Typically, gated fragments like /kæpt/, that are consistent with both the actual word and its cohort competitor facilitate the processing of both the actual target and the cohort competitor compared to unrelated control targets (Zwitserlood, 1989). These results are consistent with the idea that initial form-based access activates lexical candidates and their semantic properties, allowing facilitation of a response to a semantically related word (Marslen-Wilson, 1987).

More recently, high temporal resolution methods such as eye and hand movement studies and event-related potentials (ERP) have been used to gain more insight in the time course of competition between coactivated words. For example, a general finding in eye movement studies is that when participants view arrays of pictures while hearing a target word (in a neutral context), more fixations are made to cohort competitors of the target word than to phonologically unrelated distractors (e.g. Allopenna et al., 1998; Dahan et al., 2001; Dahan and Tanenhaus, 2004). More precisely, the amount of fixations to cohort competitors starts to diverge from fixations to unrelated distractor words as early as $200 \mathrm{~ms}$ after word onset. Taking into account that eye movements need 150-180 ms of preparation time, these data indicate that lexical activation influences eye movements in the first $100 \mathrm{~ms}$ after word onset. Importantly, the number of fixations made to targets and to cohort competitors is equal until approximately 200-300 ms after enough information is present in the signal to disambiguate targets from cohort competitors, indicating that the lexical representations of both the targets and the cohort competitors are activated and considered by participants.

Similarly, in a study in which participants were required to make a PC mouse movement towards a picture depicting a spoken word, the trajectory of the mouse movement was found to be influenced by the presence of a cohort competitor (Spivey et al., 2005). The trajectory of the mouse followed an equidistant path to both pictures for a longer time when the distractor picture was a cohort competitor. This was interpreted as being consistent with "a description of two nearly equibiased attractors initially pulling the system toward their shared midpoint" (p. 10394). Only when enough information was present in the speech signal to disambiguate the two stimuli, the target became a stronger attractor, pulling the movement of the mouse towards it.

Psychophysiological evidence that the meaning of several potential candidates is activated comes from a study by van Petten et al. (1999). In an ERP study, participants listened to sentences that ended with contextually congruous words, incongruous words that shared the same initial phonemes (cohort incongruous words), or words that rhymed with the congruous words (rhyme incongruous words). The target ERP component in this study was the N400, which is usually observed when participants are presented with semantically incongruous words in a sentence, indicating that it reflects semantic integration (Kutas and Hillyard, 1980, 1984). The critical finding in this study was that the N400 amplitude for rhyme incongruous words deviated from that of congruous words and cohort incongruous words as early as $200 \mathrm{~ms}$ before the isolation point (i.e., the moment when enough information was present to have one lexical candidate left). Moreover, the N400 amplitudes of the congruous words and the cohort incongruous words diverged from each other at a later time point, namely after the isolation point. These data clearly indicated that the meaning of alternative lexical candidates was at least partially activated and that the moment of selection depended on the amount of onset phonemes that were shared between target and competitor. These data have been replicated in a subsequent study by van den Brink et al. (2006), who also showed that the onset of the N400 effect occurs before the isolation point. Furthermore, the onset latency of the $\mathrm{N} 400$ was not influenced by the exact timing of the isolation point, which implies that semantic integration takes place before a unique word candidate has been selected.

In a cross-modal priming task, Friedrich (2005) showed that visual targets that are fully congruent with previously presented auditory primes lead to most negative ERP amplitudes at centro-temporal electrodes, followed by targets that were only partially congruent and targets that were fully incongruent, respectively. The amplitude differences caused by prime-target congruency were reflected in a P350 component which peaked at left-hemispheric scalp sites at around $350 \mathrm{~ms}$. Based on these results and those of a previous study in which the P350 was also observed in a unimodal priming task (Friedrich et al., 2004), the P350 was interpreted as an index for the activation of matching words in a modality-independent mental lexicon. In this respect, the P350 shares similarities with the M350 component observed in MEG studies (e.g., Pylkkänen et al., 2002, 2004, 2006; Stockall et al., 2004). The latency of the M350 has been found to vary with phonotactic probability of written words (Pylkkänen et al., 2002; Stockall et al., 2004). Words with high phonotactic probabilities led to lower M350 latencies than words with low probabilities. Similar to the previously mentioned P350, the M350 was interpreted to reflect lexical activation, and the decreased latency for high-probability words was a result of facilitated lexical activation.

Further evidence for the activation of multiple lexical candidates in written words came from an ERP study by Holcomb et al. (2002). Participants read words and pseudowords that belonged to small or large lexical neighborhoods while performing either a lexical decision task or a semantic categorization task. The authors found an enhanced N400 component for both words and pseudowords with a high amount of orthographic neighbors. This N400 pattern was interpreted as supportive for the view that printed strings belonging to large neighborhoods lead to increased levels of activation at either the form representation level, or the semantic representation level. Interestingly, in the semantic categorization task, effects of neighborhood size could already be observed in the 150- to 350-ms time window. Along similar lines, Barber et al. (2004) and Hutzler et al. (2004) reported an N400 component varying with syllable frequency (higher N400 for high syllable frequency stimuli), supporting the idea that high-frequency syllables trigger the activity of many lexical 
candidates since they are shared by many words. In addition to the N400 effect, and similar to Holcomb et al. (2002), an early negativity for high-frequency syllables was observed $150 \mathrm{~ms}$ (Barber et al., 2004) or 190 ms (Hutzler et al., 2004) after presentation of the stimulus. The interpretation of these results was that early first-syllable frequency effects may be associated with pre-lexical processes like phonological stimulus processing and activation of lexical candidates. In addition, Barber et al. (2004) hypothesize that the subsequent N400 effect might not just reflect differences in the activation of lexical candidates, but might also reflect differences in inhibition between lexical candidates, associated with selecting the proper candidates from many activated items.

In summary, there is general empirical evidence and consensus that speech or written input activates several initial lexical candidates matching input. For recognition to take place, it is necessary to narrow this initial set of candidates down to a single, best-fitting match. Contrary to support for coactivation of lexical candidates, the processes involved in selecting appropriate candidates have not been investigated so far. In the present study, we applied a paradigm that enabled us to look at the selection process itself, rather than the activation of candidates. In a visual gating paradigm we presented sets of three letter combinations, letter by letter (see Fig. 1).

For each set, we calculated beforehand how many lemmas in the Dutch language start with the first, the first two or all three letters of the set (CELEX database, Baayen et al., 1995). As displayed in Fig. 2, after the first letter, the cohort size (i.e., the amount of words that start with that letter) was large, the second letter reduced that size and differentiated stimuli into three types of cohorts (relatively large, medium, small), the third letter reduced the cohort size of all three conditions to the same size.

After presentation of the third letter, the amount of lexical candidates that matches current input was equal for all three groups of stimuli. However, the reduction of the cohort size (i.e., the slope that can be observed in Fig. 2) from the second

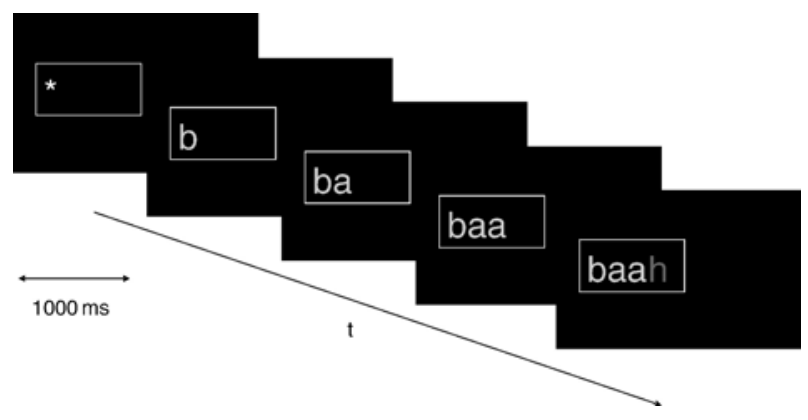

Fig. 1 - Trial display. A trial started with the presentation of a green letter (depicted as light grey in this graph) at the leftmost position in the box, on the center of the screen. The trial continued by presenting green letters at positions to the right of this letter at a rate of $1 \mathrm{~Hz}$, until a red letter appeared (depicted as the dark grey ' $h$ ' in this graph), to which the subject was asked to make a button press response. The letters on the screen never formed actual words, and the position of the red target letter was randomly varied.

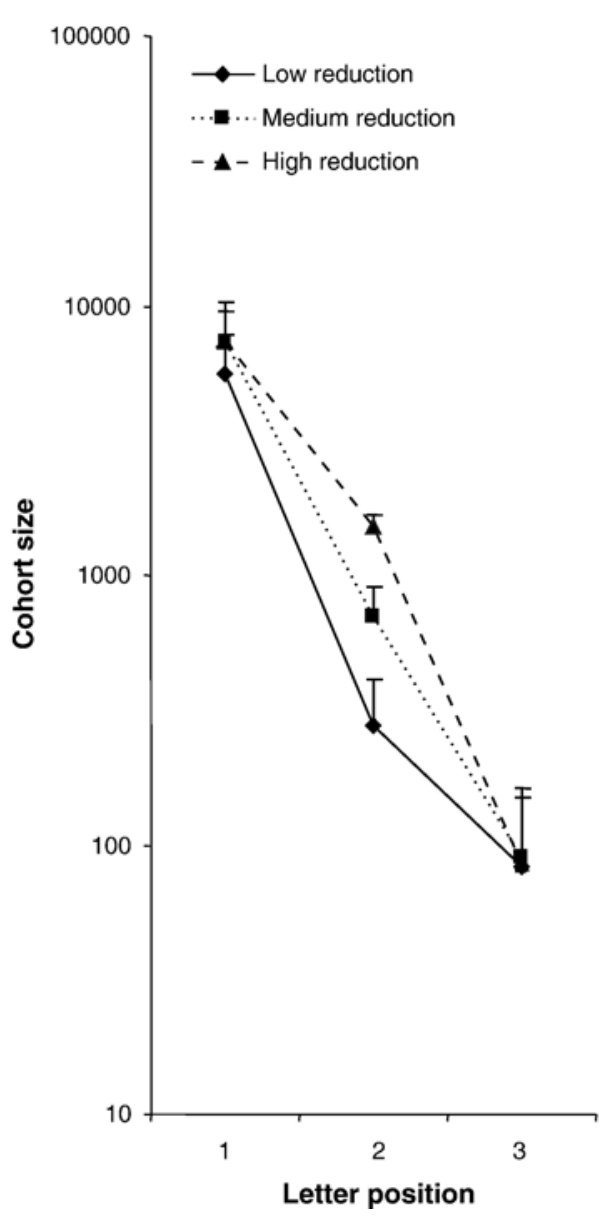

Fig. 2 - Stimulus material. Depicted are the sizes of the cohorts of words that could be formed after presentation of each of the three letters, i.e., the numbers of lemmas in Dutch that start with that letter combination. The size of the reduction of a cohort from one letter to the next is represented by the slope of the line between those two letters. Bars represent standard deviations. Please note that the $Y$-axis has a logarithmic scale.

to the third letter position differed between conditions. The rationale behind this design was that any ERP difference in response to the third letter presentation could be explained by the size of currently active cohorts, as they were similar across conditions from the onset of the third letter onwards. Rather, a variation in the ERP should reflect the selection processes that are involved in the reduction of the size of the active cohort.

The letter positions were marked in the EEG signal and the ERP signal in response to the third letter was analyzed in order to observe the reduction process at a millisecond scale. Assuming that inhibition of non-matching lexical candidates occurs at approximately the same time as the activation of the candidate set, we expected to observe effects of cohort-size reduction in similar time windows as reported for (pre-)lexical coactivation (Barber et al., 2004; Carreiras et al., 2005; Holcomb et al., 2002; Hutzler et al., 2004). Specifically, the P2 and N400 components were expected to display effects of cohort-size reduction. 


\section{Results}

\subsection{Behavioral findings}

Data reported here belong to the 16 subjects that were used in the ERP analysis. Behavioral data of 2 subjects had to be discarded due to technical problems. Inspection showed that reaction times linearly decreased with letter position. Reaction times to filler trials with the target at position 1 were $584 \mathrm{~ms}$, whereas responses to targets at position 7 took only $369 \mathrm{~ms}$. This effect of target position in filler trials was highly significant $(F(6,78)=77.76, p<0.001)$. A linear within-subjects contrast confirmed the strong linear decrease observed of reaction times as the letter position progressed $(F(1,13)=117.8$, $p<0.001)$. Since target letters never appeared on letter positions 1 to 3 in experimental trials, a comparison between experimental and filler conditions is only possible at positions 4 to 7. An ANOVA with letter position (4, 5, 6 and 7) and condition (filler vs. experimental) revealed no differences between targets that were preceded by experimental trials and those preceded by filler trials $(F(1,13)=2.15, p>0.1)$. Again, the effect of target position was highly significant $(F(3,39)=$
45.62, $p<0.001)$. There was no interaction between target position and condition $(F(3,39)=0.50, p>0.5)$.

Due to the simple nature of the task, little errors were made. The average amount of missed targets was $5 \%$ across conditions. An ANOVA on the amount of missed targets in the filler condition revealed no effect of target position $(F(6,78)=$ $1.41, p>0.1)$. Analysis of the last 4 letter positions revealed no difference between the amount of missed targets that were preceded by either experimental or filler trials $(F(1,13)=0.173$, $p>0.5)$. No effect of letter position was observed $(F(3,39)=$ $0.53, p>0.5)$, nor was there an interaction between letter position and condition $(F(3,39)=0.52, p>0.5)$. The amount of false alarms was extremely small (on average $0.5 \%$ ). There were no differences between experimental and filler conditions $(F(1,13)=1.53, p>0.1)$ or letter position $(F(5,65)=3.23$, $p>0.05)$, nor was there a significant interaction between position and condition $(F(5,65)=0.29, p>0.5)$.

\subsection{Electrophysiological findings}

\subsubsection{Third letter presentation}

The grand average waveforms of ERPs elicited by the third letter are presented in Fig. 3. All conditions showed an N1-P2

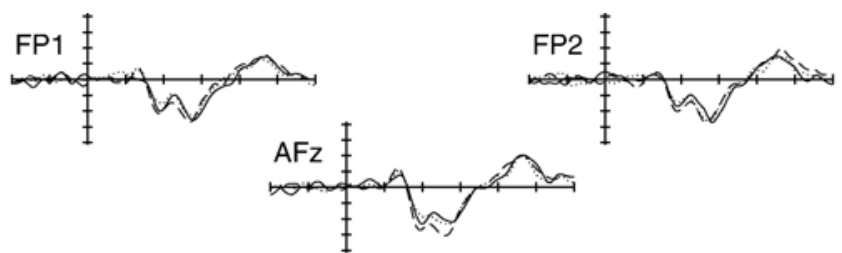

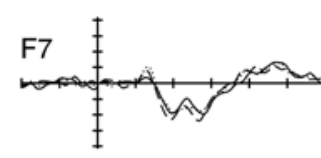
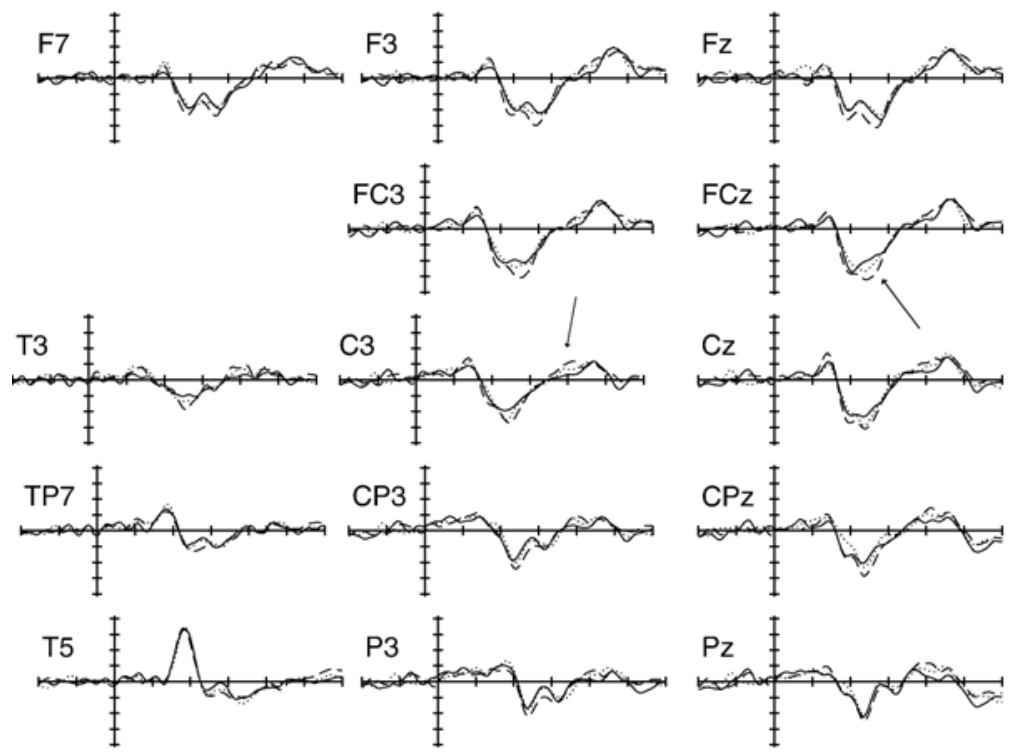

P3 f
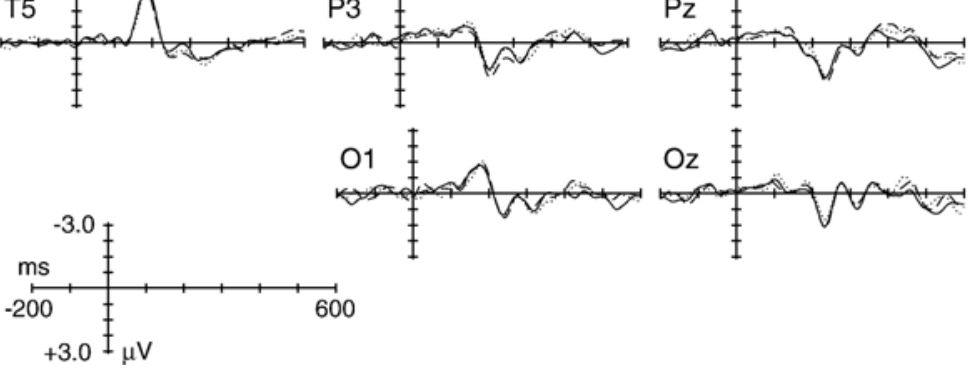
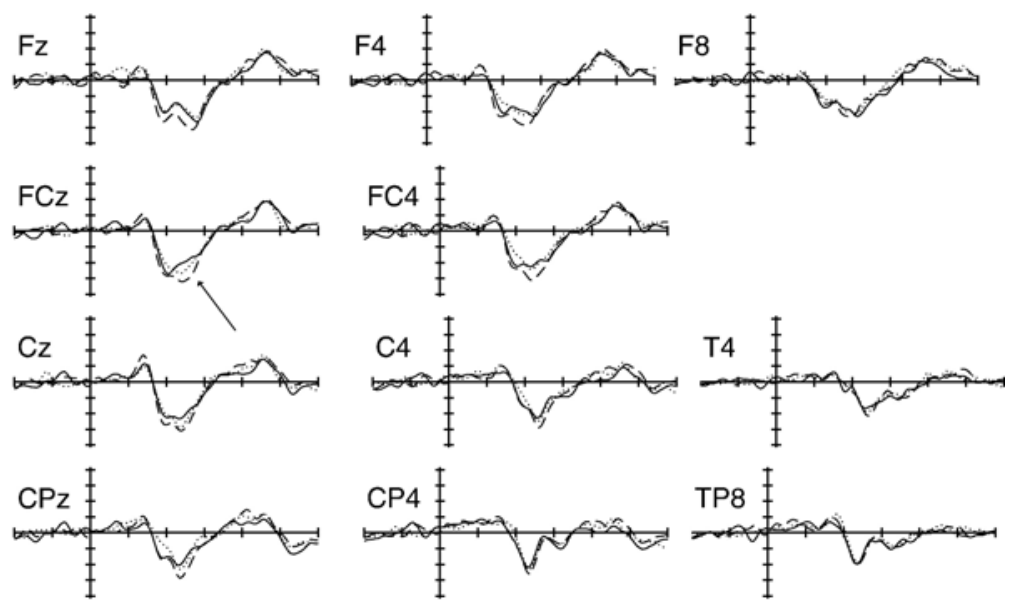

TP8
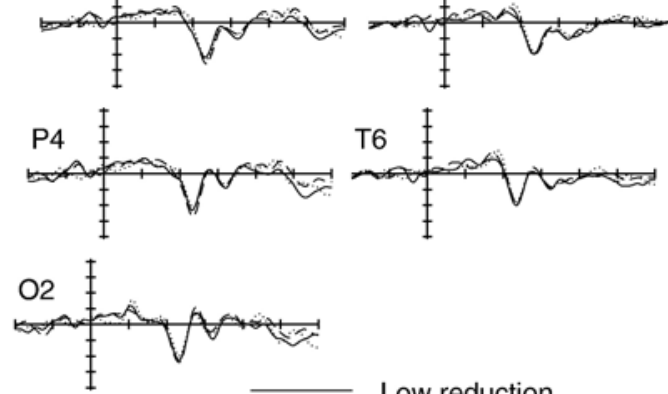

Low reduction Medium reduction High reduction

Fig. 3 - ERP waveforms in response to the presentation of the third letter. ERPs are time-locked to the presentation of the third letter ( $0 \mathrm{~ms})$, at which cohort-size reduction was manipulated, but overall cohort size was kept constant. Arrows indicate components that scaled with cohort-size reduction. 
complex which was most prominent at anterior sites, followed by a large frontal negativity occurring between 300 and $500 \mathrm{~ms}$ after onset of the third letter. At posterior sites, the morphology of the ERPs was slightly different, probably due to the nature of our stimulus material (letter-by-letter gating). The posterior N1 component was slightly delayed, compared to the anterior N1 component, and was especially large at electrodes $\mathrm{T} 5$ and $\mathrm{O} 1$ (i.e., contra-lateral to the location of the newly presented letters). The subsequent P2 component was less pronounced than at anterior sites. Instead of the frontally visible negative component between 300 and $500 \mathrm{~ms}$, a few small negative and positive going deflections are observed at posterior sites.

For a reliable determination of time windows of interest, root-mean-square (RMS) transformations (i.e., the square root of the mean of the squared potentials from each commonreferenced electrode) were calculated (see Fig. 4). Note that these RMS plots were only used for visual selection of the time windows of interest. The subsequent statistical analysis was based on the mean amplitudes of the ERPs. Visual inspection of the RMS plots clearly indicated three time windows, especially at fronto-central sites, in which the parametric cohort-size manipulation resulted in systematic variations of the RMS and ERP amplitudes. The first of these time windows was related to the N1 component, between 124 and $164 \mathrm{~ms}$. The second time window of interest included the P2 component, between 212 and 280 ms. Finally the window from 360 to $440 \mathrm{~ms}$ was selected for further analysis as it showed variation in the late negativity across conditions at central sites. Mean area amplitudes for each of these time windows were calculated for each condition for each subject, and were entered in a repeated measures General Linear Model (GLM) analysis with cohort-size reduction (3 levels: low, medium, high), laterality (3 levels: left (F3 to O1), middle (Fz to Oz), right
(F4 to O2)) and anterior-posterior (6 levels: F, FC, C, CP, P and O lines) as factors. All reported $p$-values of tests with more than one degree of freedom in the numerator were GreenhouseGeisser corrected to adjust for violations of the sphericity assumption (Greenhouse and Geisser, 1959).

Analysis of the average ERPs in the 124- to 164-ms time window showed no significant effects of cohort-size reduction $[F(2,30)=0.71, p=0.47]$. The effects of laterality $[F(2,30)=2.06$, $p=0.15]$ and anterior-posterior $[F(5,75)=0.21, p=0.70]$ were not significant either, nor were any of the interactions (all $p$ values $>0.1$ ). Inspection of the single electrodes where the effect was most apparent visually (see Figs. 3 and 5) did not reveal any effects of cohort-size reduction in this time window (all $p$-values $>0.1$ ).

For the $212-$ to $280-\mathrm{ms}$ time window, the cohort-size reduction (3) × laterality (3) xanterior-posterior (6) analysis revealed a significant main effect of cohort-size reduction $[F$ $(2,30)=3.66, p=0.045]$. The most positive mean amplitudes were observed in the high reduction condition $(1.67 \mu \mathrm{V})$, followed by the medium $(1.39 \mu \mathrm{V})$ and low reduction conditions $(1.23 \mu \mathrm{V})$, respectively. The increasing mean area amplitude with increasing cohort-size reduction was confirmed by a significant linear within-subjects contrast for the reduction factor $[F(1,15)=5.24, p=0.037]$, supporting the observation of a linear relationship between the experimental manipulation and ERP amplitude in this time window. A significant main effect of the anterior-posterior factor was observed $[F(4,60)=$ 21.84, $p<0.001]$. Mean amplitudes were largest over central sites and declined towards more anterior and posterior sites. The main effect of laterality was not significant $[F(2,30)=1.93$, $p=0.18]$. There was a statistical trend towards an interaction between cohort-size reduction and the anterior-posterior factor $[F(10,150)=2.53, p=0.064]$. Post hoc analysis of the cohort-size reduction effect at the different anterior-posterior

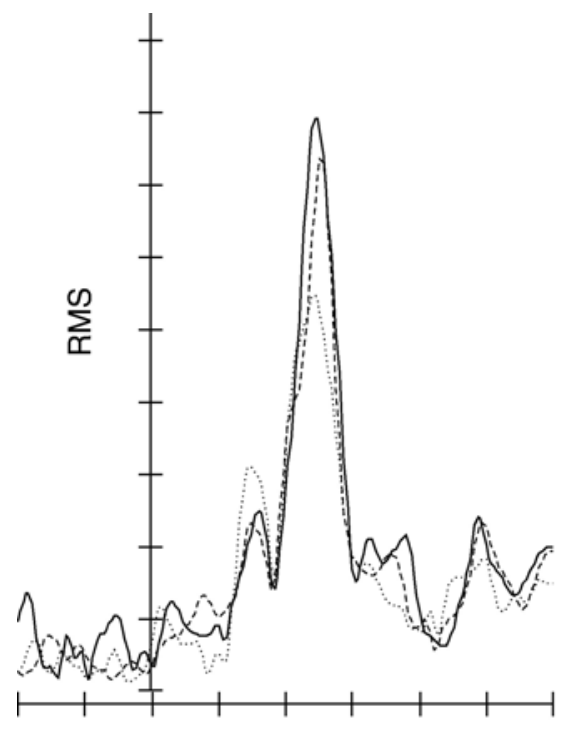

Position 1

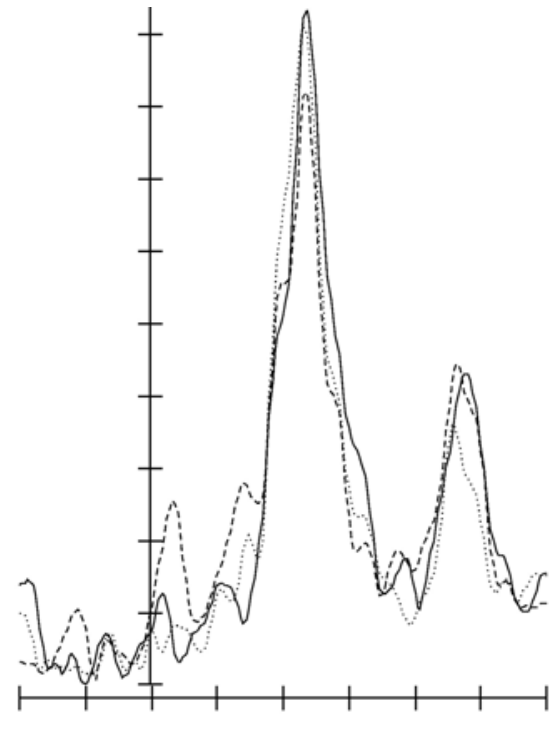

Position 2

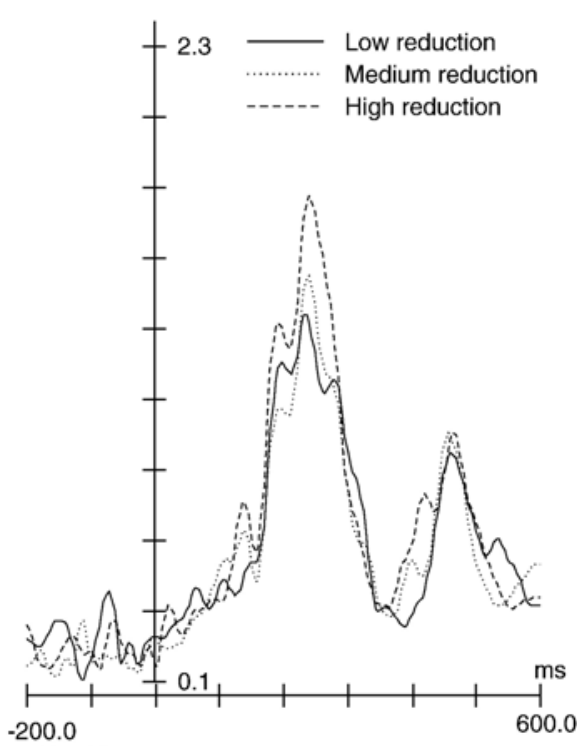

Position 3

Fig. 4 - RMS plots of each letter position. RMS plots were calculated by taking the square root of the average of the squared values of each of the 29 common-referenced electrodes. RMS values at the third letter position (rightmost figure) were used to define three time windows for further analysis: the first one ranging from 124 to $164 \mathrm{~ms}$, a second one from 212 to $280 \mathrm{~ms}$, and a third one from 360 to $400 \mathrm{~ms}$. All subsequent analyses were performed on the ERP values per subject per condition. 

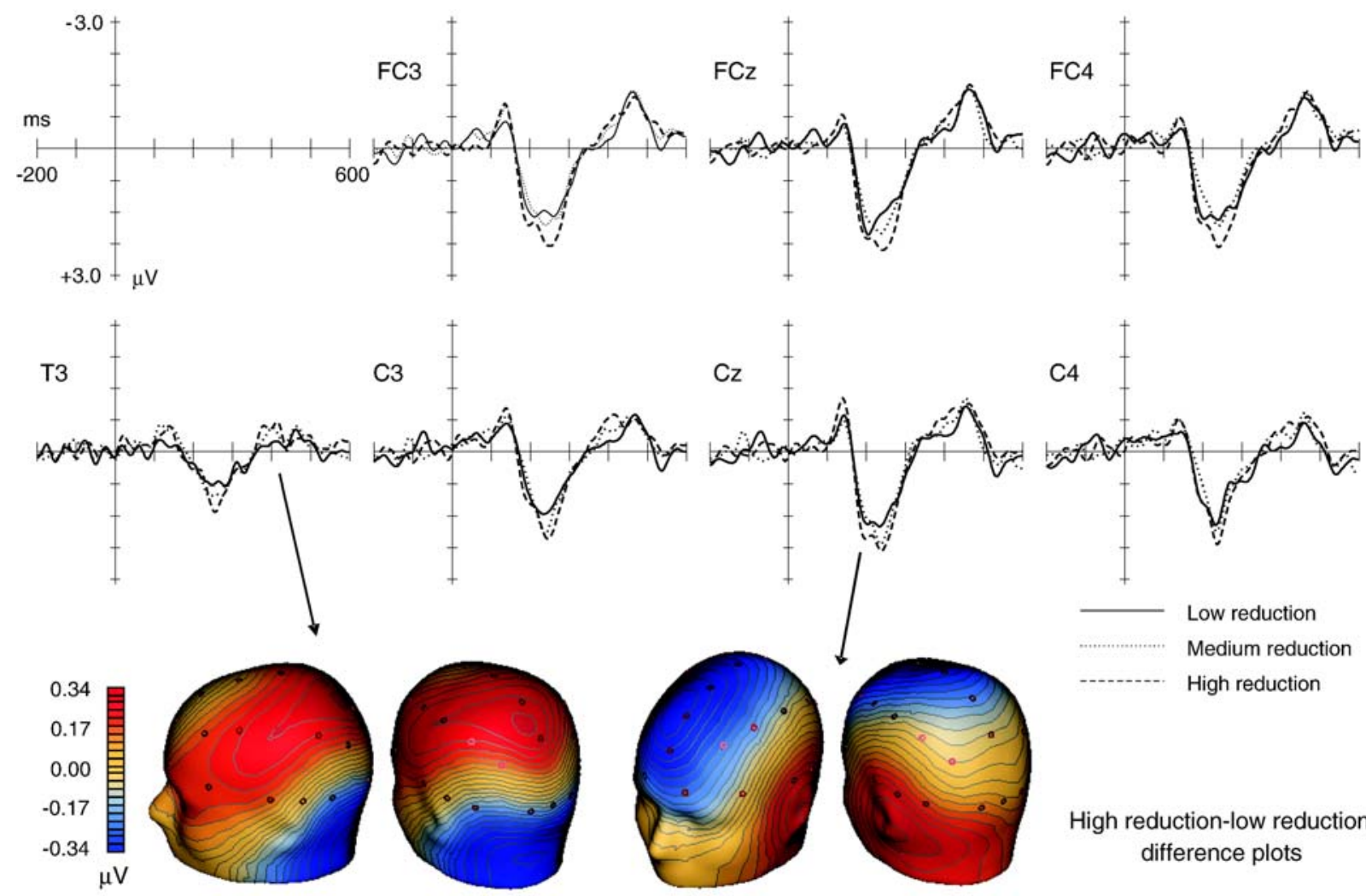

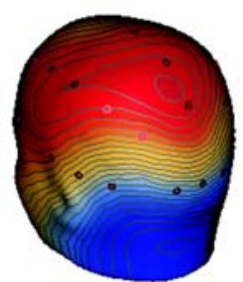

Late negativity

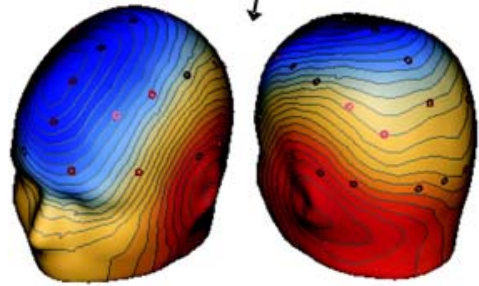

P2

Fig. 5 - ERP waveforms and topographical difference maps in response to the presentation of the third letter. Waveforms are magnified versions of electrodes shown in Fig. 3 at which cohort-size reduction effects could be observed. Topographic maps represent the difference between the high reduction and low reduction conditions in the N400 time window (late negativity, left side) and the P2 time window (right side).

levels revealed that the effect was strongest at F and FC lines, slowly declining towards more posterior sites. None of the other interactions were significant (all $p$-values $>0.1$ ).

For the 360- to 440-ms time window, a cohort-size reduction (3) $\times$ laterality (3) ×anterior-posterior (6) GLM revealed a significant main effect of cohort-size reduction $[F(2,30)=3.89$, $p=0.047]$. The most negative mean amplitudes were observed in the high reduction condition $(-0.54 \mu \mathrm{V})$, followed by the medium $(-0.35 \mu \mathrm{V})$ and low $(-0.15 \mu \mathrm{V})$ conditions. Furthermore, a significant laterality effect was observed $[F(2,30)=6.65$, $p=0.005]$, as well as a significant effect of the anterior-posterior factor $[F(4,60)=3.74, p=0.038]$. Mean amplitudes were higher at midline electrodes than at more lateral electrodes and increased at more anterior sites. None of the interaction terms were significant (all $p$-values $>0.05$ ). The topography of the difference between the high and low reduction group in this time window (see Fig. 5) indicated a left central-temporal focus of the effect. The linear within-subjects contrast was significant $[F(1,15)=5.19, p=0.038]$, confirming the linear relationship between cohort-size reduction and ERP amplitude in this time window at these scalp sites.

\subsubsection{First letter presentation}

In the 124- to 164-ms time window, no effects of stimulus condition were observed in response to the presentation of the first letter $[F(2,30)=2.79, p>0.1]$. There was a significant effect of the anterior-posterior factor $[F(5,75)=6.61, p=0.01]$; highest amplitudes were observed at FC and $C$ lines. The laterality effect was not significant, nor were any of the interactions (all $p$-values $>0.1)$.

In the 218- to 280-ms time window, a significant effect of condition was observed $[F(2,30)=5.20, p=0.022]$. Most positive mean amplitudes were observed in the 'low reduction' condition $(2.02 \mu \mathrm{V})$, followed by the 'high' $(1.55 \mu \mathrm{V})$ and 'medium' $(1.22 \mu \mathrm{V})$ conditions. There was also a significant main effect of laterality $[F(2,30)=8.29, p=0.002]$, with less positive mean amplitudes at right-hemisphere leads. No other main effects or interactions were observed in this time window (all $p$-values $>0.1$ ).

In the 360- to $440-\mathrm{ms}$ time window, a significant laterality effect $[F(2,30)=11.19, p<0.001]$ and an interaction of this factor with the anterior-posterior factor $[F(10,150)=4.04, p=0.003]$ were observed. None of the other main factors or interaction terms approached significance (all $p$-values $>0.2$ ).

\subsubsection{Second letter presentation}

In the 124- to 164-ms time window, there was a marginally significant effect of stimulus condition in response to the presentation of the second letter $[F(2,30)=3.44, p=0.062]$. Amplitudes in the group of stimuli that had the highest reduction at letter position 3 were slightly higher than those of the other conditions. There was a main effect of laterality 
$[F(2,30)=4.08, p=0.046]$, with higher amplitudes for left hemisphere electrodes. The unexpected effect of condition in this time window seemed mainly due to a problem in the baseline at left-lateralized electrodes for this condition, which is reflected by a significant condition $\times$ laterality interaction $[F(4,60)=3.13, p=0.039]$. More detailed analysis confirmed that the effect of condition was only significant over left hemisphere leads $[F(2,30)=6.43, p=0.008]$. At these leads, the 'high reduction' group differs from the other two conditions $(p<0.05)$. No other significant interactions were observed in this time window.

In the 212- to 280-ms time window, a significant effect of laterality $[F(2,30)=7.98, p<0.002]$ and of the anterior-posterior factor $[F(5,75)=7.50, p=0.012]$ were observed. There was no main effect of condition $[F(2,30)=0.96, p>0.25]$, nor were there any significant interactions (all $p$-values $>0.25$ ).

In the 360- to 440-ms time window, a significant effect of condition was observed $[F(2,30)=3.66, p=0.05]$. Most negative mean amplitudes were observed for the 'high reduction' $(-0.35 \mu \mathrm{V})$ condition, followed by the 'medium' $(-0.07 \mu \mathrm{V})$ and 'low' $(0.05 \mu \mathrm{V})$ conditions. The linear within-subjects contrast for this factor was significant as well $[F(1,15)=5.49$, $p=0.033]$. Furthermore, there was a significant effect of laterality $[F(2,30)=9.04, p=0.001]$, with more negative mean amplitudes for central electrodes. There was also a significant interaction between laterality and the anterior-posterior factor $[F(10,150)=2.96, p=0.02]$; the effect of laterality was slightly stronger at fronto-central electrode locations. None of the other factors or interactions approached significance (all $p$-values $>0.25)$.

\section{Discussion}

In this study, a new paradigm was applied to investigate neural processes of cohort-size reduction during word recognition. By visually presenting parts of words letter by letter at a slow rate during ERP recording, we were able to investigate parametrically manipulated cohort reduction online. The cohort-size reduction was manipulated by creating groups of stimuli in which the amount of lexical candidates that did not match the input after the presentation of the third letter differed between conditions. The aim of the study was to relate ERP effects to cohort reduction or selection processes proper. The potentially confounding factor of cohort size was controlled for by keeping it constant at the letter position of interest (i.e., position 3). Previous studies on cohort effects in language comprehension found evidence for a coactivation of lexical candidates both behaviorally (e.g., Allopenna et al., 1998; Dahan et al., 2001; Dahan and Tanenhaus, 2004; MarslenWilson, 1987; Spivey et al., 2005; Zwitserlood, 1989) and electrophysiologically (Barber et al., 2004; Holcomb et al., 2002; Hutzler et al., 2004). The latter identified two ERP components of interest (the P2 and N400 components) whose amplitudes systematically varied with the amount of activated lexical items. Granted the assumption that coactivation of lexical candidates is closely related to the processes involved in inhibiting non-matching candidates we hypothesized that these ERP components are possible target components for detecting effects of cohort-size reduction as well. In fact, in the present study ERPs in response to the third letter scaled with cohort-size reduction in the time windows of the P2 and N400 components. In these time windows, higher ERP amplitudes were observed in conditions where cohort-size reduction was high and vice versa. Since higher ERP amplitudes are usually associated with higher neural processing loads (e.g., Hagen et al., 2006; Handy and Mangun, 2000; Swaab et al., 2004; van Petten and Luka, 2006) these data support the view that stronger inhibitive processes are required when larger groups of lexical candidates are discarded.

\subsection{P2 time window}

Interestingly, effects of cohort-size reduction were observed as early as the frontal P2 component (212-280 ms), a time window in which orthographic neighborhood size effects (Holcomb et al., 2002) and syllable frequency effects (Barber et al., 2004; Hutzler et al., 2004) have been reported. Our results confirm that the ERP in this window is also sensitive to the specific process of cohort reduction. The P2 effect observed in the preceding studies was interpreted as a precursor for the subsequent N400 effect, caused by a different amount of activated candidates. In our design, we dissociated the reduction of candidates from the amount of active candidates, and kept the amount of active lexical candidates identical across conditions after presentation of the third letter. Since we too observed a variation in P2 amplitude, we suggest that it reflects the amount of inhibition of words that mismatch phonological/orthographic input, rather than the amount of activated lexical candidates. The observed positive correlation between P2 amplitude and size of reduction implies that inhibition is stronger in situations where the cohort of activated lexical candidates has to be reduced to a larger degree.

This interpretation is supported by a variety of studies. In several different paradigms, frontal positive components have been identified that were involved in inhibitory and selective processes. For example, in the visual domain, a component variously named Frontal Selection Positivity (FSP), anterior P2 (P2a) or Frontal Positivity (FP) has been linked to stimulus evaluation and target selection (Kenemans et al., 1993; Potts, 2004; Smid et al., 1999), or to the suppression of irrelevant features (Guillem et al., 2001). In the auditory domain, the P2 component has been interpreted as being involved in the inhibition of distractors (Melara et al., 2002) and in target detection (Potts et al., 1998). Although the exact nature of this P2 component is still under debate (see Potts, 2004), most authors stress its involvement in the evaluation of task relevance or in the inhibition of responses to distractor stimuli. The $\mathrm{P} 2$ component can be seen as reflecting inhibition and/or selection in a more general, modality-independent manner. From the cited literature, the frontal positivity was reported as being sensitive to the selection of visual features like orientation, color, shape, location and even faces. In addition, it has also been related to the selection of auditory stimuli, or - as reported here - to the linguistic reduction of a lexical cohort. Alternatively, a family of P2s could reflect very modality specific inhibition/selection. Depending on the type of information (visual, auditory, linguistic, or non-linguistic) one would expect slight variations in the P2 across tasks. The 
present study cannot give an answer on the functional specificity of the P2. Future studies are needed to systematically compare the $\mathrm{P} 2$ topography and the underlying neural generator/s across tasks. Most importantly, whether general or specific, the observed P2 in the present paradigm speaks of the time course of cohort reduction, which takes place in the 212- to 280-ms time window after letter onset.

Recent studies have found evidence that lexical access is well underway between 100 and $200 \mathrm{~ms}$ after presentation of visual words (Hauk et al., 2006a; Sereno and Rayner, 2003). In this time window, effects of lexical frequency (Hauk and Pulvermuller, 2004; Hauk et al., 2006a; Sereno et al., 1998, 2003), syllable frequency (Barber et al., 2004; Hutzler et al., 2004), biand trigram frequency (Hauk et al., 2006b) and neighborhood size (Holcomb et al., 2002) have been observed, indicating that lexical access must have already taken place. The effects of cohort-size reduction observed in the present study occurred slightly later than in these studies, i.e., slightly after $200 \mathrm{~ms}$, even though most of the word has already been processed when the target letter appears. A possible explanation is that, in the current study, the target letter did not complete an actual word: each three-letter string was still only a partial word, congruent with many candidates. The fact that no single candidate is selected, may have caused a delay in the lexical access process. Alternatively, the effects presently observed take place slightly after the initial lexical access stage. That is, although candidates receive activation in an earlier time window, the actual process of inhibiting nonmatching candidates does not start until shortly after the initial activation. Future research should address this issue, for example by manipulating reduction and frequency effects simultaneously in order to investigate the time courses of both effects.

It is also important to note that the direction of the cohortsize reduction effect we observed in the P2 time window is reversed in comparison with the effects of syllable frequency and neighborhood size reported in previous studies (Barber et al., 2004; Holcomb et al., 2002; Hutzler et al., 2004). That is, we observed more positive amplitudes in conditions where lots of inhibition takes place, whereas previous results showed less positive amplitudes for conditions where many items were activated. In this respect, it can be informative to have a look at the results at letter positions 1 and 2 . The results at the first letter presentation replicated previously reported results. That is, P2 amplitudes in response to the presentation of the first letter were less positive for letters with which many words can be formed, which is in line with previous results where highfrequency first syllables and stimuli with many orthographic neighbors lead to less positive P2 amplitudes. Comparing results at letter position 1 with those at position 3 confirms that two effects with opposing directions are taking place in the P2 time window: initial activation of candidates decreases P2 amplitude, whereas inhibition of candidates that do not match input anymore increases it. Extra support for this observation comes from the results at position 2. At this position, the condition with the lowest amount of possible candidates was also the condition with the lowest amount of cohort-size reduction compared to position 1. In other words: at letter position 2, the counteracting effects of candidate activation and inhibition should lead to a null-effect on P2 amplitude. Indeed, no significant difference between conditions could be observed in the P2 amplitude, nor did the effect approach significance. The opposing direction of the effects of activation and inhibition raises the important question on what the strength of each of these effects is. It also implies that previously observed P2 effects are actually smaller than they would have been if the inhibition factor would have been kept constant. The design of the current study is not suitable for estimating the magnitude of each effect. A design including a condition in which cohort-size reduction is kept constant but cohort activation is parametrically varied would yield valuable additional information.

\subsection{N400 time window}

The second selection-related effect was observed in the later negative component, at left temporal sites. This negativity scaled with cohort-size reduction in a similar manner as the P2 effect. The distribution of the effect in this time window (left temporal focus) and the duration of the effect (360-440 ms after onset of the third letter) imply that the observed component is not a "standard" N400, which is usually characterized as having a centro-parietal distribution slightly biased towards the right hemisphere 300-600 ms after onset of the critical stimulus (Kutas and van Petten, 1994). The function of the $\mathrm{N} 400$ is related to semantic integration within sentences (Kutas and Hillyard, 1984) and across sentences and discourse processing (van Berkum et al., 1999). The N400 has also been reported in the auditory domain as being sensitive to both context-based (top-down) and acoustic phonetic (bottom-up) information (van den Brink et al., 2006; van Petten et al., 1999), indicating its involvement in lexical access. There are several reasons why we did not observe a "standard" N400. For example, in our visual gating paradigm, we looked into the integration at a letter-by-letter manner and it could well be that a letter-by-letter integration is reflected in a relatively "smaller" N400. Another reason for the lack of a standard N400 could be that we manipulated neither the amount of active lexical items nor the difficulty of integrating the current input into a context. The amount of lexical candidates was equal between conditions after presentation of the third letter, and letter strings were presented without a semantic context. Furthermore, our subjects were never asked to zoom into single lexical items because the cohort size of the three-letter word fragments was always bigger than one.

Nevertheless, the effect observed in the present study temporally and morphologically overlaps with the early window of the "standard" N400 effect. As reported above, these early stages of the N400 are likely to be involved in phonological stimulus analysis (van den Brink et al., 2006; van Petten et al., 1999). We suggest that our observed N400like effect reflects integration processes in the mental lexicon. More specifically, given the subjects' task to process pseudowords on a letter-by-letter basis, we suggest that the effect reflects the updating of the cohort of active candidates in the mental lexicon. This update involves the adjustment of the cohort from second to third letter input, which entails the inhibition of non-matching items that were activated by the preceding partial input. The relatively late time window in which the update occurs (N400, 360-440 ms after onset) 
suggests that it is a consequence of the relatively early inhibitory process (P2, 212-280 ms after third letter onset).

As with the P2 time window, it might be informative to look at effects in the N4 time window at letter positions 1 and 2. In contrast to the $\mathrm{P} 2$, effects of cohort-size reduction in the current study are in the same direction as effects of syllable frequency and neighborhood frequency in previous studies in this time window. Thus we should observe effects of cohort activation and inhibition at all three letter positions. Interestingly, no effects of condition were observed at letter position 1. An explanation for this is that no update of the cohort of active candidates is required after presentation of the first letter. If the early N400 time window indeed reflects the update of the activated lexical candidates as more information becomes available, then effects of cohort size should only be expected after an initial cohort of candidates has been activated by previously presented information. This was the case only after presentation of letters two and further, hence the absence of any differences in this time window at letter position 1 . In line with this interpretation, effects of condition were observed in this time window after presentation of the second letter, indicating that lexical processing is taking place. Observed amplitudes were lowest for the 'low' condition where cohort size and cohort reduction (from position 1 to 2) were smallest, followed by the 'medium' and the 'high' group, respectively. Since the 'medium' group had the highest amount of reduction from position 1 to 2, but the 'high' group had the highest cohort size at position 2, this implicates that the effect of cohort size in this time window has a bigger effect on ERP amplitude than does cohort reduction. However, since our study was designed to test for inhibition effects at position 3, not activation effects at other positions, better controlled studies should be employed in the future to investigate the strength of both effects.

Stimuli were presented in an unnatural manner during this experiment. With a few exceptions such as letter-by-letter message displays at animated advertisement boards, at car radio fronts, or with text messaging at mobile phones, people do not often encounter written stimuli that are presented to them in an incremental fashion in daily life. Generally, words are fixated once before the next word is processed. Although a single fixation is enough to extract the visual information about a written word, fixation times are vulnerable to factors such as word length, frequency and familiarity (Sereno and Rayner, 2003), and some models of written language comprehension suggest that lexical access in written words takes place in a left-to-right fashion (e.g., Coltheart et al., 2001; Johnson and Pugh, 1994). In line with this idea, word recognition experiments showed that words with an early Orthograpic Uniqueness Point (OUP, the visual analog of the Uniqueness Point that is used in the auditory gating paradigm) are recognized faster than those with a late OUP (Coltheart and Rastle, 1994; Kwantes and Mewhort, 1999; Lindell et al., 2005; Rastle and Coltheart, 1999). Thus, it seems reasonable to assume that at least in some situations lexical access in response to written words proceeds in a left-to-right manner. Of course, we cannot claim that the effects we observed with the current paradigm reflect lexical mechanisms involved in every real-world situation. But by putting time constraints on the availability of orthographic information at the visual input level, as in the current study, rather than within the language processing system, as is generally the case, a time window was created that allowed investigation of the processes involved in cohort-size reduction, and specifically the inhibitory processes associated with this.

In summary, this study has revealed two ERP components related to cohort-size reduction, a factor that has received little attention in the research literature before. We observed a relatively early component (P2) that, we suggest, reflects the neural correlate of the inhibition of candidates based on phonological/orthographic information, followed by a component that mirrors the neural processes involved in updating the active candidates in the lexicon (N400 time window). Given the similarity of the time windows of the present study (cohort-size reduction) and other studies (activation of matching candidates) our data support the plausible assumption that activation of lexical candidates and inhibition of nonmatching candidates are closely related in time. In line with others (Barber et al., 2004; Holcomb et al., 2002; Hutzler et al., 2004), our results show that these two phases in the word recognition process can be manipulated separately, and that they independently affect different ERP components.

\section{Experimental procedures}

\subsection{Participants}

Twenty-two right-handed native speakers of Dutch participated in this study. They were paid for their time. All were neurologically healthy and had normal or corrected to normal vision. Data of six participants had to be discarded due to excessive amounts of eye movement artefacts (more than $25 \%$ of trials), or technical problems. The data of the remaining 16 subjects ( 5 females, mean age 22.8 years) were analyzed. This research project was approved by the ethical committee of the Faculty of Psychology, Maastricht University.

\subsection{Stimuli}

In order to keep frequency and cohort-size constant, while manipulating cohort-size reduction, three groups of 36 threeletter combinations were created. For each of these threeletter combinations, the amount of lexical candidates (lemmas) in the Dutch language that, start with the first, the first two, or all three letters was calculated (Celex database for lemma frequencies Baayen et al., 1995). For example, Dutch has over 5300 lemmas that start with the letter ' $h$ ', about 1500 lemmas start with 'ho', and 200 candidates with 'hon'. Based on these stimulus properties, three groups of stimuli were created that differed in the size of the cohort of possible alternatives after presentation of a new letter (see Fig. 2). Groups were created in such a way that the average cohort size after presentation of the first letter was 5593 (standard deviation 2961), 7347 (3017) and 7356 (2153), for the three groups. More importantly, after presentation of the second letter, these cohorts were reduced to 276 (97), 704 (208) and 1526 (139) words for the three groups, labeled Low, Medium and High, respectively. The critical factor in the design was the equal size of the cohorts after the presentation of the third 
letter; 83 (60), 90 (73) and 93 (65), respectively. Based on this material, the size of the reduction of cohort size (i.e., cohort size after the second letter minus cohort size after the third letter) differentiates the three groups. That is, the size of the reduction was 193 for the Low condition, 614 for the Medium condition, and 1442 for the High condition. To control for frequency effects, the average frequency of each of the members of the cohorts was matched for two-letter combinations (122 (84), 130 (63), 131 (90), respectively) and for threeletter combinations (103 (84), 98 (68), 102 (88), respectively).

Letters were presented in green (RGB $0 ; 255 ; 0)$ or in red (RGB $255 ; 0 ; 0$, target for the reaction task of the subject, see below). Font type was Courier, letter size was $0.5^{\circ}$ of visual angle. Letters were sequentially presented on a black background. The first letter appeared at the location of the fixation cross, subsequent letters were presented at $0.6^{\circ}$ of visual angle to the right of the previous letter, with a maximum of seven letters. The letters on the screen never formed a real word, and the red letter never appeared in the first three letters of an experimental trial. In addition to potential word segments, filler letter sequences were included that were presented in the same manner as the relevant strings, except that they were created by randomly generating strings of letters. These filler trials were included to distract participants from the fact that many of the stimuli formed parts of words, in order to reduce anticipation effects for word meaning in incoming strings. Furthermore, in these filler trials targets could also appear at the first 3 letter positions, which insured subjects' attention to all letter positions.

\subsection{Procedure}

Participants were told that they participated in a simple reaction time experiment. After explanation of the task, and application of the electrodes, participants were comfortably seated in an electrically shielded, sound-attenuated room in front of a computer monitor. They were told to keep the amount of movement as low as possible, and to be as fast and accurate as possible on the reaction task. On the monitor, a black screen with a white box was projected. In this box, a fixation cross appeared for a variable amount of time (1900-2500 ms), to which participants were asked to fixate. A trial sequence reflecting the letter-by-letter gating paradigm is displayed in Fig. 1. A trial started with the disappearance of the fixation cross, which was substituted by a green letter. At a rate of $1 \mathrm{~Hz}$, letters were added to the right of this letter until a red letter appeared. The participants' task was to monitor the color of the newly presented letters, and press a response button on a response box with their right index finger whenever a red letter appeared, which ended the trial. A fixation cross reappeared until a new trial started. The 108 experimental stimuli (36 trigram trials $\times 3$ conditions) were randomly alternated with 54 filler stimuli. This block of stimuli was repeated three times per subject, leading to a total of 108 trials per condition.

\subsection{EEG recording}

The electroencephalogram (EEG) was measured using 29 tin electrodes mounted in an elastic cap (QuickCap, Neuroscan Inc. Tm). The electrodes were positioned according to the international 10-20 system (Jasper, 1958). Electrodes Fz, F3, F4, F7, F8, FCz, FC3, FC4, FP1, FP2, AFz, T3, T4, T5, T6,TP7, TP8, $\mathrm{Cz}, \mathrm{C} 3, \mathrm{C} 4, \mathrm{CPz}, \mathrm{CP} 3, \mathrm{CP} 4, \mathrm{Pz}, \mathrm{P} 3, \mathrm{P} 4, \mathrm{Oz}, \mathrm{O} 1, \mathrm{O} 2$ were measured as active leads. Scalp sites were online referenced to an electrode placed at the left mastoid (A1). The signal of an additional electrode placed at the right mastoid (A2), referenced to A1, was later used for offline re-referencing of the signal to the average of A1 and A2. Vertical eye movements and blinks were measured with a bipolar montage placed above and below the left upper and lower orbital ridge. Horizontal eye movements were recorded with a bipolar montage placed at the left and right canthi. All electrode impedances were kept below $5 \mathrm{~K} \Omega$. The EEG was amplified (Synamp, Neuroscan Inc.) with a $0.05-30 \mathrm{~Hz}$ bandpass and sampled at $2.5-\mathrm{ms}$ intervals $(400 \mathrm{~Hz})$ and stored to disk. Epochs of $800 \mathrm{~ms}$ were created, time-locked to the onset of the third letter in each trial. Epochs included a 200-ms prestimulus baseline correction interval and they were 1-30 Hz bandpass filtered (Butterworth, zero phase, 24dB/oct). After removal of eye blinks and other artifacts (on average $10 \%$ of the data, no difference between conditions), ERPs were calculated separately for each subject and each condition.

\section{Acknowledgments}

This project was supported by NWO ASPASIA grant 015.001.053 to B.M. Jansma (Schmitt). The authors would like to thank Boukje Habets, Dirk Köster and Ann-Kathrin Bröckelmann for their valuable discussion.

\section{REFEREN C ES}

Allopenna, P.D., Magnuson, J.S., Tanenhaus, M.K., 1998. Tracking the time course of spoken word recognition using eye movements: evidence for continuous mapping models. J. Mem. Lang. 38, 419-439.

Ans, B., Carbonnel, S., Valdois, S., 1998. A connectionist multiple-trace memory model for polysyllabic word reading. Psychol. Rev. 105, 678-723.

Baayen, R.H., Piepenbrock, R., Gulikers, L., 1995. The CELEX Lexical Database (Release 2) [CD-ROM] (Philadelphia, PA, Linguistic Data Consortium, University of Pennsylvania).

Barber, H., Vergara, M., Carreiras, M., 2004. Syllable-frequency effects in visual word recognition: evidence from ERPs. NeuroReport 15, 545-548.

Carreiras, M., Vergara, M., Barber, H., 2005. Early event-related potential effects of syllabic processing during visual word recognition. J. Cogn. Neurosci. 17, 1803-1817.

Coltheart, M., Rastle, K., 1994. Serial processing in reading aloud: evidence for dual-route models of reading. J. Exp. Psychol. Hum. Percept. Perform. 20, 1197-1211.

Coltheart, V., Avons, S.E., Masterson, J., Laxon, V.J., 1991. The role of assembled phonology in reading comprehension. Mem. Cogn. 19, 387-400.

Coltheart, M., Rastle, K., Perry, C., Langdon, R., Ziegler, J., 2001. DRC: a dual route cascaded model of visual word recognition and reading aloud. Psychol. Rev. 108, 204-256.

Cutler, A., Clifton, C.E., 1999. Comprehending spoken language: a blueprint of the listener. In: Brown, C.M., Hagoort, P. (Eds.), The Neurocognition of Language Processing. Oxford University Press, Oxford, pp. 123-166. 
Dahan, D., Tanenhaus, M.K., 2004. Continuous mapping from sound to meaning in spoken-language comprehension: immediate effects of verb-based thematic constraints. J. Exp. Psychol. Learn. Mem. Cogn. 30, 498-513.

Dahan, D., Magnuson, J.S., Tanenhaus, M.K., 2001. Time course of frequency effects in spoken-word recognition: evidence from eye movements. Cogn. Psychol. 42, 317-367.

Friedrich, C.K., 2005. Neurophysiological correlates of mismatch in lexical access. BMC Neurosci. 6, 64.

Friedrich, C.K., Kotz, S.A., Friederici, A.D., Gunter, T.C., 2004. ERPs reflect lexical identification in word fragment priming. J. Cogn. Neurosci. 16, 541-552.

Gaskell, M.G., Marslen-Wilson, W.D., 1997. Integrating form and meaning: a distributed model of speech perception. Lang. Cogn. Processes 12, 613-656.

Goldinger, S.D., Luce, P.A., Pisoni, D.B., Marcario, J.K., 1992. Form-based priming in spoken word recognition: the roles of competition and bias. J. Exp. Psychol. Learn. Mem. Cogn. 18, 1211-1238.

Greenhouse, S., Geisser, S., 1959. On methods in the analysis of profile data. Psychometrika 24, 95-112.

Guillem, F., Bicu, M., Debruille, J.B., 2001. Dissociating memory processes involved in direct and indirect tests with ERPs to unfamiliar faces. Cogn. Brain Res. 11, 113-125.

Hagen, G.F., Gatherwright, J.R., Lopez, B.A., Polich, J., 2006. P3a from visual stimuli: task difficulty effects. Int. J. Psychophysiol. $59,8-14$.

Handy, T.C., Mangun, G.R., 2000. Attention and spatial selection: electrophysiological evidence for modulation by perceptual load. Percept. Psychophys. 62, 175-186.

Hauk, O., Pulvermuller, F., 2004. Effects of word length and frequency on the human event-related potential. Clin. Neurophysiol. 115, 1090-1103.

Hauk, O., Davis, M.H., Ford, M., Pulvermuller, F., Marslen-Wilson, W.D., 2006a. The time course of visual word recognition as revealed by linear regression analysis of ERP data. NeuroImage 30, 1383-1400.

Hauk, O., Patterson, K., Woollams, A., Watling, L., Pulvermuller, F., Rogers, T.T., 2006b. [Q:] When would you prefer a SOSSAGE to a SAUSAGE? [A:] At about 100 msec. ERP correlates of orthographic typicality and lexicality in written word recognition. J. Cogn. Neurosci. 18, 818-832.

Holcomb, P.J., Grainger, J., O'Rourke, T., 2002. An electrophysiological study of the effects of orthographic neighborhood size on printed word perception. J. Cogn. Neurosci. 14, 938-950.

Hutzler, F., Bergmann, J., Conrad, M., Kronbichler, M., Stenneken, P., Jacobs, A.M., 2004. Inhibitory effects of first syllable-frequency in lexical decision: an event-related potential study. Neurosci. Lett. 372, 179-184.

Jasper, H.H., 1958. The ten-twenty electrode system of the international federation in electroencephalography and clinical neurophysiology. EEG J. 10, 371-375.

Johnson, N.F., Pugh, K.R., 1994. A cohort model of visual word recognition. Cogn. Psychol. 26, 240-346.

Kenemans, J.L., Kok, A., Smulders, F.T., 1993. Event-related potentials to conjunctions of spatial frequency and orientation as a function of stimulus parameters and response requirements. Electroencephalogr. Clin. Neurophysiol. 88, 51-63.

Kutas, M., Hillyard, S.A., 1980. Reading senseless sentences: brain potentials reflect semantic incongruity. Science 207, 203-205.

Kutas, M., Hillyard, S.A., 1984. Brain potentials during reading reflect word expectancy and semantic association. Nature 307, 161-163.

Kutas, M., van Petten, C., 1994. Psycholinguistics electrified. Event-related brain potential investigations. In: Gernsbacher, M.A. (Ed.), Handbook of Psycholinguistics. Academic Press, San Diego, CA.
Kwantes, P.J., Mewhort, D.J.K., 1999. Evidence for sequential processing in visual word recognition. J. Exp. Psychol. Hum. Percept. Perform. 25, 376-381.

Lindell, A.K., Nicholls, M.E., Kwantes, P.J., Castles, A., 2005. Sequential processing in hemispheric word recognition: the impact of initial letter discriminability on the OUP naming effect. Brain Lang. 93, 160-172.

Marslen-Wilson, W.D., 1987. Functional parallelism in spoken word-recognition. Cognition 25, 71-102.

Masson, M.E.J., 1995. A distributed memory model of semantic priming. J. Exp. Psychol. Learn. Mem. Cogn. 21, 3-23.

McClelland, J.L., Elman, J.L., 1986. The TRACE model of speech perception. Cogn. Psychol. 18, 1-86.

Melara, R.D., Rao, A., Tong, Y., 2002. The duality of selection: excitatory and inhibitory processes in auditory selective attention. J. Exp. Psychol. Hum. Percept. Perform. 28, 279-306.

Norris, D., McQueen, J.M., Cutler, A., 1995. Competition and segmentation in spoken-word recognition. J. Exp. Psychol. Learn. Mem. Cogn. 21, 1209-1228.

Perfetti, C., 1999. Comprehending written language: a blueprint of the reader. In: Brown, C.M., Hagoort, P. (Eds.), The Neurocognition of Language Processing. Oxford University Press, Oxford, pp. 167-208.

Potts, G.F., 2004. An ERP index of task relevance evaluation of visual stimuli. Brain Cogn. 56, 5-13.

Potts, G.F., Dien, J., Hartry-Speiser, A.L., McDougal, L.M., Tucker, D.M., 1998. Dense sensor array topography of the event-related potential to task-relevant auditory stimuli. Electroencephalogr. Clin. Neurophysiol. 106, 444-456.

Pylkkänen, L., Stringfellow, A., Marantz, A., 2002. Neuromagnetic evidence for the timing of lexical activation: an MEG component sensitive to phonotactic probability but not to neighborhood density. Brain Lang. 81, 666-678.

Pylkkänen, L., Feintuch, S., Hopkins, E., Marantz, A., 2004. Neural correlates of the effects of morphological family frequency and family size: an MEG study. Cognition 91, B35-B45.

Pylkkänen, L., Llinas, R., Murphy, G.L., 2006. The representation of polysemy: MEG evidence. J. Cogn. Neurosci. 18, 97-109.

Rastle, K., Coltheart, M., 1999. Serial and strategic effects in reading aloud. J. Exp. Psychol. Hum. Percept. Perform. 25, 482-503.

Sereno, S.C., Rayner, K., 2003. Measuring word recognition in reading: eye movements and event-related potentials. Trends Cogn. Sci. 7, 489-493.

Sereno, S.C., Rayner, K., Posner, M.I., 1998. Establishing a time-line of word recognition: evidence from eye movements and event-related potentials. NeuroReport 9, 2195-2200.

Sereno, S.C., Brewer, C.C., O'Donnell, P.J., 2003. Context effects in word recognition: evidence for early interactive processing. Psychol. Sci. 14, 328-333.

Smid, H.G., Jakob, A., Heinze, H.J., 1999. An event-related brain potential study of visual selective attention to conjunctions of color and shape. Psychophysiology 36, 264-279.

Spivey, M.J., Grosjean, M., Knoblich, G., 2005. Continuous attraction toward phonological competitors. Proc. Natl. Acad. Sci. U. S. A. 102, 10393-10398.

Stockall, L., Stringfellow, A., Marantz, A., 2004. The precise time course of lexical activation: MEG measurements of the effects of frequency, probability, and density in lexical decision. Brain Lang. 90, 88-94.

Swaab, T.Y., Camblin, C.C., Gordon, P.C., 2004. Electrophysiological evidence for reversed lexical repetition effects in language processing. J. Cogn. Neurosci. 16, 715-726.

van Berkum, J.J., Hagoort, P., Brown, C.M., 1999. Semantic integration in sentences and discourse: evidence from the N400. J. Cogn. Neurosci. 11, 657-671.

van den Brink, D., Brown, C.M., Hagoort, P., 2006. The cascaded nature of lexical selection and integration in auditory sentence processing. J. Exp. Psychol., Learn. Mem. Cogn. 32, 364-372. 
van Petten, C., Luka, B.J., 2006. Neural localization of semantic context effects in electromagnetic and hemodynamic studies. Brain Lang. 97, 279-293.

van Petten, C., Coulson, S., Rubin, S., Plante, E., Parks, M., 1999.

Time course of word identification and semantic integration in spoken language. J. Exp. Psychol. Learn. Mem. Cogn. 25, 394-417.

Zwitserlood, P., 1989. The locus of the effects of sentential-semantic context in spoken-word processing. Cognition 32, 25-64. 